\title{
ON ZANELLO'S LOWER BOUND FOR GENERIC QUOTIENTS OF LEVEL ALGEBRAS
}

\author{
JONAS SÖDERBERG
}

(Communicated by Irena Peeva)

\begin{abstract}
We give a shorter and more straightforward proof of a theorem of Zanello on lower bounds for Hilbert functions of generic level quotients of artinian level algebras.
\end{abstract}

The notion of a generic level quotient, also known as a relatively compressed level algebra, has been used in the study of level algebras. Zanello gave a generalization of a result of Iarrobino on lower bounds for Hilbert functions of generic level quotients. In this note we give a shorter and more straightforward proof of Zanello's result.

We consider graded artinian $k$-algebras $A=R / I$, where $R=k\left[x_{1}, x_{2}, \ldots, x_{n}\right]$ is the standard graded polynomial ring in $n$ variables over an infinite field $k$ and $I$ is a homogeneous ideal of $R$. The socle of an artinian algebra $A$ is the homogeneous ideal

$$
\operatorname{Soc}(A)=\left\{f \in A \mid x_{i} f=0 \text { for } i=1,2, \ldots, n\right\},
$$

and furthermore $A$ is a level algebra of type $t$ if $A$ is artinian, $\operatorname{Soc}(A)$ is concentrated in a single degree and $\operatorname{dim}_{k} \operatorname{Soc}(A)=t$. For any algebra $A$ and any graded $A$-module $M$ the annihilator of $M$ is the ideal $\operatorname{Ann}_{A}(M)=\{f \in A \mid f M=0\}$.

The dual of an artinian algebra is the graded $R$-module $A^{\vee}=\bigoplus_{u} \operatorname{Hom}_{k}\left(A_{u}, k\right)$ with multiplication defined by $f \varphi(g)=\varphi(f g)$ for any $f \in R, g \in A$ and $\varphi \in$ $\bigoplus_{u} \operatorname{Hom}_{k}\left(A_{u}, k\right)$. The grading of $A^{\vee}$ is given by $A_{u}^{\vee}=\operatorname{Hom}_{k}\left(A_{-u}, k\right)$. For any graded $A$-module, $M$, and integer, $c$, we denote by $M(c)$ the graded module with grading given by $M(c)_{u}=M_{c+u}$.

The Hilbert function of a graded $R$-module $M$ is the function $\mathbb{Z} \rightarrow \mathbb{Z}$ given by $u \mapsto \operatorname{dim}_{k} M_{u}$.

Let $A$ be a level algebra. By a generic level quotient of type $s$ of $A$ we mean a level quotient of type $s$ with, degree by degree, maximal Hilbert function among all level quotients of type $s$. Note that such a maximal Hilbert function exists when $k$ is an infinite field because of the upper semi-continuity of the Hilbert function. Zanello has the following in [3].

Theorem 1 (Zanello). Let $A=A_{0} \oplus A_{1} \oplus \cdots \oplus A_{c}$ be a graded artinian level $k$ algebra of type $t$, where $k$ is an infinite field, and furthermore let $H_{u}^{s}=\operatorname{dim}_{k} A_{u}^{(s)}$,

Received by the editors May 29, 2011 and, in revised form, September 2, 2012.

2010 Mathematics Subject Classification. Primary 13A02.

Key words and phrases. Graded modules, level algebras, Hilbert functions. 
where $A^{(s)}$ is a generic level quotient of $A$ of type s. Then

$$
H_{u}^{s} \geq \frac{1}{t^{2}-1}\left((t s-1) H_{u}^{t}+(t-s) H_{c-u}^{t}\right) .
$$

Iarrobino [1, Theorem 2.4] proves this for $s=2$, although he states it differently. Zanello's proof uses a complicated induction argument with the result of Iarrobino as the induction base. We now present another proof starting with a more direct generalization of the idea of Iarrobino.

Consider a graded $R$-module $M$ and a submodule $M^{\prime}$ generated by $s$ elements of degree zero. We say that $M^{\prime}$ is generic if $M^{\prime}$ has, degree by degree, maximal Hilbert function among all submodules of $M$ generated by $s$ elements of degree zero. Again, these maximal Hilbert functions exist if the field $k$ is infinite.

Proposition 2. Assume that $k$ is an infinite field. Let $M$ be a graded $R$-module minimally generated by $t$ elements of degree zero, and let $A=R / \operatorname{Ann}_{R}(M)$. Furthermore, let $B$ be a generic submodule of $M$ generated by a single element of degree zero. Then we have

$$
\operatorname{dim}_{k} B_{u} \geq \frac{1}{t+1}\left(\operatorname{dim}_{k} A_{u}+\operatorname{dim}_{k} M_{u}\right),
$$

for each integer $u$. In particular, if $A^{\prime}$ is a level algebra with socle in degree $c$ and we take $M$ to be $M=\left(A^{\prime}\right)^{\vee}(-c)$, we get

$$
\operatorname{dim}_{k} B_{u} \geq \frac{1}{t+1}\left(\operatorname{dim}_{k} A_{u}^{\prime}+\operatorname{dim}_{k} A_{c-u}^{\prime}\right)
$$

for each integer $u$.

Proof. Being linearly independent is an open condition, so we may choose a $k$ basis $e_{1}, e_{2}, \ldots, e_{t}$ of $M_{0}$, the graded component of degree zero of $M$, such that the submodule generated by $e_{i}$ is generic for $i=1,2, \ldots, t$ and the same is true for the submodule generated by $w=\sum_{i=1}^{t} e_{i}$. Then $B$ has the same Hilbert function as the submodule generated by $w$, so we may assume that $B$ is this submodule.

The complex

$$
0 \rightarrow \operatorname{Ann}_{A}(w) \stackrel{\varphi}{\rightarrow} \bigoplus_{i=1}^{t} A e_{i} \stackrel{\psi}{\rightarrow} M \rightarrow 0
$$

where the map $\varphi$ is given by $g \mapsto\left(g e_{1}, g e_{2}, \ldots, g e_{t}\right)$, is exact at all places except in the middle. To see this, first note that $\operatorname{Ker} \varphi=\operatorname{Ann}_{A}(M)$ and that $\operatorname{Ann}_{A}(M)=0$ since $A=R / \operatorname{Ann}_{R}(M)$, so $\varphi$ is injective. The image of $\varphi$ is in the kernel of $\psi$ since $\psi(\varphi(g))=g e_{1}+\cdots+g e_{t}=g w=0$ and $\psi$ is surjective since $e_{1}, e_{2}, \ldots, e_{t}$ generate $M$. From the complex above we get

$$
\begin{aligned}
\operatorname{dim}_{k} \operatorname{Ann}_{A}(w)_{u} \leq \operatorname{dim}_{k}(\operatorname{Ker} \psi)_{u} & =\sum_{i=1}^{t} \operatorname{dim}_{k} A_{u} e_{i}-\operatorname{dim}_{k} M_{u} \\
& =t \operatorname{dim}_{k} B_{u}-\operatorname{dim}_{k} M_{u} .
\end{aligned}
$$

Now $B \cong A / \operatorname{Ann}_{A}(w)$ and we get

$$
\operatorname{dim}_{k} B_{u}=\operatorname{dim}_{k} A_{u}-\operatorname{dim}_{k} \operatorname{Ann}(w)_{u} \geq \operatorname{dim}_{k} A_{u}-t \operatorname{dim}_{k} B_{u}+\operatorname{dim}_{k} M_{u},
$$

which gives the first inequality. For the second inequality note that $M=\left(A^{\prime}\right)^{\vee}(-c)$ is generated in degree zero and that

$$
A=R / \operatorname{Ann}_{R}(M) \cong A^{\prime}
$$


since $\operatorname{Ann}_{R}(M)=\operatorname{Ann}_{R}\left(A^{\prime}\right)$. Now, since $\operatorname{dim}_{k}\left(\left(A^{\prime}\right)^{\vee}(-c)\right)_{u}=\operatorname{dim}_{k} A_{c-u}^{\prime}$, the second inequality follows from the first one.

Proposition 3. Let $M$ be a graded $R$-module minimally generated in degree zero by $t$ elements. Let $M^{(s)}$ be a generic submodule of $M$ minimally generated by $s$ elements of degree zero for each $s=1,2, \ldots, t$ and let $H_{u}^{s}=\operatorname{dim}_{k} M_{u}^{s}$. Then the sequence $H_{u}^{1} \leq H_{u}^{2} \leq \cdots \leq H_{u}^{t}=\operatorname{dim}_{k} M_{u}$ is convex, that is,

$$
H_{u}^{s} \geq \frac{1}{2}\left(H_{u}^{s-1}+H_{u}^{s+1}\right) .
$$

This means that $H_{u}^{s}$ must be above or on the line through $H_{u}^{1}$ and $H_{u}^{t}$, that is,

$$
H_{u}^{s} \geq \frac{1}{t-1}\left((t-s) H_{u}^{1}+(s-1) H_{u}^{t}\right) .
$$

Proof. Let $M$ be generated by $e_{1}, \ldots, e_{t}$ and assume that these generators are general enough so that any subset of these generators generates a generic submodule. Then, for any $s$, the inclusion

$$
R e_{1} \cap \sum_{i=2}^{s} R e_{i} \subseteq R e_{1} \cap \sum_{i=2}^{s+1} R e_{i}
$$

gives the surjection

$$
\begin{array}{ccc}
R e_{1} /\left(R e_{1} \cap \sum_{i=2}^{s} R e_{i}\right) & \rightarrow R e_{1} /\left(R e_{1} \cap \sum_{i=2}^{s+1} R e_{i}\right) \rightarrow 0 \\
\sum_{i=1}^{s} R e_{i} / \sum_{i=2}^{s} R e_{i} & \sum_{i=1}^{s+1} R e_{i} / \sum_{i=2}^{s+1} R e_{i}
\end{array}
$$

Since, for any $r$,

$$
\operatorname{dim}_{k}\left(\sum_{i=1}^{r} R e_{i} / \sum_{i=2}^{r} R e_{i}\right)_{u}=H_{u}^{r}-H_{u}^{r-1},
$$

the surjection yields

$$
H_{u}^{s}-H_{u}^{s-1} \geq H_{u}^{s+1}-H_{u}^{s}
$$

which gives the first inequality of the proposition.

Proof of Theorem 1. We have that $A^{\vee}(-c)$ is a graded module minimally generated by $t$ elements of degree zero, and $A^{(s)^{\vee}}(-c)$ is a generic submodule of $A^{\vee}(-c)$ generated by $s$ elements of degree zero. From the second inequality of Proposition 3 we get

$$
H_{u}^{s} \geq \frac{1}{t-1}\left((t-s) H_{u}^{1}+(s-1) H_{u}^{t}\right) .
$$

From the second inequality of Proposition 2 and the equalities $\operatorname{dim}_{k} B_{u}=H_{u}^{1}$, $\operatorname{dim}_{k} A_{u}=H_{u}^{t}$ we get

$$
H_{u}^{1} \geq \frac{1}{t+1}\left(H_{c-u}^{t}+H_{u}^{t}\right)
$$

Combining the two inequalities above gives the inequality

$$
\begin{aligned}
H_{u}^{s} & \geq \frac{1}{t-1}\left(\frac{t-s}{t+1}\left(H_{c-u}^{t}+H_{u}^{t}\right)+(s-1) H_{u}^{t}\right) \\
& =\frac{1}{t^{2}-1}\left((t s-1) H_{u}^{t}+(t-s) H_{c-u}^{t}\right) .
\end{aligned}
$$


Remark 4. Note that the lower bound of the second equation of Proposition 3 doesn't always give a sequence that satisfies the first, so the first inequality is stronger. For example, if $t=11, H_{u}^{1}=1$ and $H_{u}^{7}=6$, then we get Figure 1 , where the dots indicate the lower bound of the second inequality and the circles the lower bound of the first. The slope of the line is

$$
k_{u}=\frac{H_{u}^{t}-H_{u}^{1}}{t-1}
$$

so if we draw a line through the point $\left(1, H_{u}^{1}\right)$ with slope $\left\lceil k_{u}\right\rceil$ and another line through the point $\left(t, H_{u}^{t}\right)$ with slope $\left\lfloor k_{u}\right\rfloor$, we see that the lower bound of the first inequality is given by the smallest values on the union of these two lines, that is,

$$
H_{u}^{s} \geq \min \left\{\left\lceil k_{u}\right\rceil s+H_{u}^{1},\left\lfloor k_{u}\right\rfloor(s-t)+H_{u}^{t}\right\} .
$$

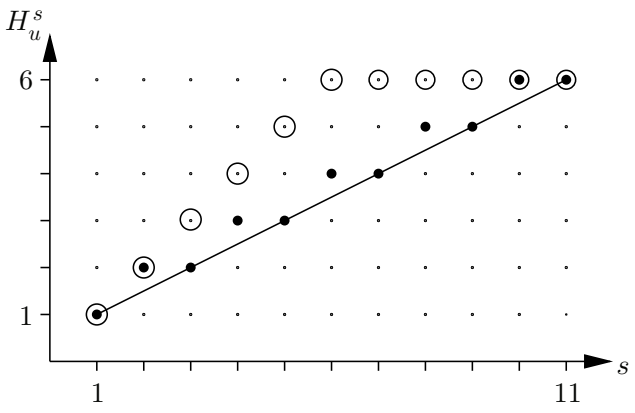

FiguRE 1. Lower bound for $t=11, H_{u}^{1}=1$ and $H_{u}^{7}=6$

Remark 5. This article was written in 2006 but never submitted for publication apart from being in the author's dissertation. Dan Laksov 2 recently found and corrected a mistake in the key linear algebra lemma used by Zanello [3] in his original proof of the bound. This makes the author's paper more important, in that it contains the first entirely correct proof of Zanello's bound. Notice also that Laksov's paper considerably generalizes the results of Zanello and the author.

\section{REFERENCES}

[1] Anthony Iarrobino, Hilbert functions of Gorenstein algebras associated to a pencil of forms, Projective varieties with unexpected properties, Walter de Gruyter, Berlin, 2005, pp. 273-286. MR2202259 (2006i:13030)

[2] Dan Laksov, On Zanello's lower bound for level algebras, Proc. Amer. Math. Soc. 141 (2013), no. 5, 1519-1527, DOI 10.1090/S0002-9939-2012-11427-3. MR3020839

[3] Fabrizio Zanello, Partial derivatives of a generic subspace of a vector space of forms: quotients of level algebras of arbitrary type, Trans. Amer. Math. Soc. 359 (2007), no. 6, 2675-2686 (electronic), DOI 10.1090/S0002-9947-07-04015-9. MR2286051 (2007k:13034)

Department of Mathematics, KTH, S-100 44 Stockholm, Sweden

Current address: Baggensgatan 23, 11131 Stockholm, Sweden

E-mail address: jonassod@gmail.com 